\title{
Adding twist to anisotropic fluids
}

\author{
J. P. Krisch and E. N. Glass ${ }^{\mathrm{a})}$ \\ Department of Physics, University of Michigan, Ann Arbor, Michigan 48109
}

(Received 18 November 2001; accepted for publication 17 December 2001)

\begin{abstract}
We present a solution generating technique for anisotropic fluids which preserves specific Killing symmetries. Anisotropic matter distributions that can be used with the one parameter Ehlers-Geroch transform are discussed. Example space-times that support the appropriate anisotropic stress-energy are found and the transformation applied. The $3+1$ black string solution is one of the space-times with the appropriate matter distribution. Use of the transform with a black string seed is discussed. (C) 2002 American Institute of Physics. [DOI: 10.1063/1.1448683]
\end{abstract}

\section{INTRODUCTION}

Physically relevant solution generating techniques were developed in the 1960s and 1970s. Ehlers, ${ }^{1}$ Harrison, ${ }^{2}$ and Geroch $^{3,4}$ showed that a projective transform on the norm and twist $(\lambda, \omega)$, of a Killing vector will generate a Killing vector with norm and twist $\left(\lambda^{\prime}, \omega^{\prime}\right)$. Starting with a vacuum space-time and a twist-free Killing vector, their method adds twist. For example, their method applied to the vacuum Schwarzschild metric generates a NUT metric. The generating method can be applied to any vacuum space-time with a Killing vector and has been generalized to the Einstein-Maxwell spaces ${ }^{5}$ and to some matter space-times. ${ }^{6}$ The extension to matter metrics is restrictive; Stephani ${ }^{7}$ has shown that the only two equations of state that can be treated within this formalism are

$$
\begin{gathered}
\rho=P, \\
\rho+3 P=0 .
\end{gathered}
$$

Raca and Zsigrai ${ }^{8}$ have also considered solution generating on fluids with this equation of state. The result clearly applies to fluids with isotropic stress. A close examination of the method used to generate the allowed matter distributions shows that it can be generalized to fluids with anisotropic stresses.

There has been increasing interest in general relativistic systems with anisotropic stress. Herrera and $\operatorname{Santos}^{9}$ have reviewed some of the possible causes and the related general relativistic solutions. Anisotropic fluid spheres have been a useful model for discussing anisotropy since the early work of Bowers and Liang ${ }^{10}$ on anisotropic fluid spheres. More recently Corchero ${ }^{11}$ has discussed a post-Newtonian approximation to anisotropic fluid spheres. Das et al. ${ }^{12}$ and Das and Kloster ${ }^{13}$ have investigated the spherically symmetric collapse of anisotropic fluid objects into a black hole. Hernandez, Lunez, and Percoco ${ }^{14}$ have treated anisotropy and nonlocal equations of state in radiating spheres. Conformally flat anisotropic spheres were examined by Herrera et al. ${ }^{15}$ Glass and Krisch ${ }^{16}$ have discussed diffusion induced anisotropies in a Vaidya atmosphere. Following up the recent interest in dimensional effects, Harko and $\mathrm{Mak}^{17}$ discussed charged anisotropic fluid spheres in $D$-dimensions. Anisotropy in cosmological solutions has also been studied by McManus and Coley, ${ }^{18}$ vandenHoogen and Coley, ${ }^{19}$ Giovannini, ${ }^{20}$ and Rainsford. ${ }^{21}$ Relative motion as a source of anisotropy in multifluid systems was suggested long ago by Jeans. ${ }^{22}$

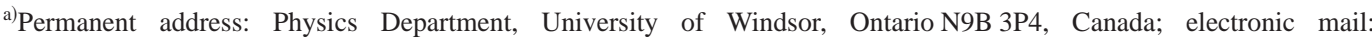
englass@umich.edu
} 
Letelier ${ }^{23}$ has shown that two perfect fluids in relative motion can be described as a system with anisotropic pressures and has given the standard two-fluid stress energy form.

Physically, anisotropy is finding increasing application in systems at the density extremes of very compact objects and very diffuse mass clusters. This increasing interest in fluid solutions with anisotropic pressures suggests it would be of interest to extend the solution generating technique to space-times that have anisotropic fluid content. While it is relatively simple to create anisotropic fluid solutions by changing the functional dependence of metric potentials, the generating technique here will preserve specific Killing symmetries while obtaining new anisotropic solutions.

In Sec. II, we briefly review the formalism that leads to the isotropic pressure restrictions on the standard solution generating technique. The extension to anisotropic stresses follows directly from this. Isotropic seed space-times are discussed in Sec. III. Some examples of space-times containing the allowed anisotropies are in Sec. IV. Killing symmetries for the possible anisotropic spaces are covered in Sec. V, and Sec. VI has a discussion of the effect of the Geroch transformation on the fluid description in these spaces.

\section{FORMALISM}

\section{A. The Killing description}

Let $\left(M, g_{a b}\right)$ be a solution of the Einstein field equations with energy density $\rho$ and isotropic pressure $P$. Assume that $g_{a b}$ has a Killing vector $\xi^{a}$ with norm $\lambda$ and twist $\omega^{a}$ where

$$
\begin{gathered}
\lambda:=\xi^{a} \xi_{a}, \\
\omega^{a}:=\epsilon^{a b c d} \xi_{b} \nabla_{c} \xi_{d} .
\end{gathered}
$$

The induced metric on the three-dimensional space of vectors orthogonal to the Killing vector is

$$
h_{a b}=g_{a b}-\xi_{a} \xi_{b} / \lambda .
$$

The vacuum field equations can be written in terms of $\lambda, \omega$ and $\gamma_{a b}$,

$$
\begin{gathered}
\gamma_{a b}=|\lambda| h_{a b}, \\
\omega_{a}=D_{a} \omega,
\end{gathered}
$$

where $\gamma_{a b}$ is conformally related to $h_{a b},{ }^{3} \omega$ is the scalar potential associated with the Killing twist, and $D_{a}$ is the covariant derivative for metric $\gamma_{a b}$.

\section{B. Vacuum space-times}

Consider the action written in the conformal three-space of $\gamma_{a b}$,

$$
I=\int d^{3} x \sqrt{\gamma}\left[\mathcal{R}-\frac{1}{2 \lambda^{2}}\left(D_{a} \lambda D^{a} \lambda+D_{a} \omega D^{a} \omega\right)\right] .
$$

A projective transform of the complex potential $\tau=\omega+i \lambda$ can be written

$$
\tau^{\prime}=\frac{\tau \cos (\delta)+\sin (\delta)}{\cos (\delta)-\tau \sin (\delta)},
$$

where $\delta$ is a transformation parameter. The action is invariant under this transformation, so we have added twist to the Killing vector with $\gamma_{a b}$ unchanged and can generate the new $3+1$ space-time, $g_{a b}^{\prime}$, from the formalism. The development of the transformation method is described in Refs. 3 and 4. We briefly review the transformation method for twist-free Killing vectors. 


\section{Generating the new space-time from a twist-free seed}

The generation method as described by Geroch ${ }^{3,4}$ for vacuum will be the same in the matter space-times. Starting with metric $g_{a b}$, define forms $\alpha_{a}, \beta_{a}$, and $\eta_{a}$ based on a seed Killing vector, $\xi_{a}$, with norm $\lambda$ and transformation parameter $\delta$,

$$
\begin{gathered}
\beta_{a}=\xi_{a}\left(\lambda-\lambda^{-1}\right), \\
2 \alpha_{[b, a]}=\varepsilon_{a b c d} \nabla^{c} \xi^{d}, \\
\eta_{a}=\lambda^{-1} \xi_{a}+\alpha_{a} \sin (2 \delta) .
\end{gathered}
$$

The new metric is then given by

$$
g_{a b}^{\prime}=F\left(g_{a b}-\lambda^{-1} \xi_{a} \xi_{b}\right)+(\lambda / F) \eta_{a} \eta_{b}
$$

with

$$
F=\cos ^{2}(\delta)+\lambda^{2} \sin ^{2}(\delta) .
$$

The norm of the Killing vector becomes

$$
\lambda^{\prime}=\lambda / F .
$$

The scalar twist potential that has been added to the Killing vector is

$$
\omega^{\prime}=\frac{\sin (\delta) \cos (\delta)\left(1-\lambda^{2}\right)}{F} .
$$

These are the equations that will generate the new space-time from static seed metrics.

\section{ISOTROPIC MATTER DESCRIPTIONS}

\section{A. Isotropic model 1}

To find the isotropic matter space-times that can be used with this method, consider the action $^{24}$

$$
I=\int \mathrm{d}^{3} x \sqrt{\gamma}\left[\mathcal{R}-\frac{D_{a} \lambda D^{a} \lambda}{2 \lambda^{2}}+\Psi\right]
$$

with $\Psi$ a specified function. Stephani ${ }^{7}$ has shown that the Ricci tensor in four dimensions is

$$
R_{a b}=-\Psi|\lambda|\left(g_{a b}-\xi_{a} \xi_{b} / \lambda\right)
$$

and clearly the action will be invariant under the projective transform on $\tau$. In the isotropic case, this Ricci tensor is associated with a perfect fluid for $\xi^{a}$ timelike. The fluid has an equation of state $\rho+3 P=0$, with $8 \pi \rho=3 \Psi \lambda / 2$.

\section{B. Isotropic model 2}

A second action that one can consider is ${ }^{24}$

$$
I=\int \mathrm{d}^{3} x \sqrt{\gamma}\left[\mathcal{R}-\frac{D_{a} \lambda D^{a} \lambda}{2 \lambda^{2}}-s_{a} s^{a}\right]
$$

with 


$$
s_{a} \xi^{a}=0
$$

The four-dimensional Ricci tensor for this action is

$$
R_{a b}=s_{a} s_{b}
$$

which, in the isotropic case, describes a perfect fluid if the Killing vector is spacelike. The fluid has density and pressure

$$
\begin{gathered}
8 \pi \rho=-s^{a} s_{a} / 2, \\
P=\rho .
\end{gathered}
$$

Solution generating with both of these isotropic forms has been examined by Garfinkle, Glass, and Krisch. $^{25}$

\section{ANISOTROPIC MATTER DESCRIPTION}

The form of the Ricci tensor will be the same for fluids with nonisotropic stress. The stressenergy content can be written as

$$
(1 / 8 \pi) T_{a b}=\rho u_{a} u_{b}+p_{1} e_{a}^{(1)} e_{b}^{(1)}+p_{2} e_{a}^{(2)} e_{b}^{(2)}+p_{3} e_{a}^{(3)} e_{b}^{(3)},
$$

where $\left(u^{a}, e_{a}^{(i)}, i=1,2,3\right)$ is a convenient orthogonal tetrad. The associated Ricci tensor is

$$
\begin{gathered}
R_{a b}=T_{a b}-(T / 2) g_{a b}, \\
(1 / 8 \pi) R_{a b}=\rho u_{a} u_{b}+p_{1} e_{a}^{(1)} e_{b}^{(1)}+p_{2} e_{a}^{(2)} e_{b}^{(2)}+p_{3} e_{a}^{(3)} e_{b}^{(3)}+\left(\frac{\rho-p_{\Sigma}}{2}\right) g_{a b},
\end{gathered}
$$

where $p_{\Sigma}=p_{1}+p_{2}+p_{3}$.

\section{A. Anisotropic model 1}

Consider Ricci tensor $R_{a b}=-\Psi|\lambda|\left(g_{a b}-\xi_{a} \xi_{b} / \lambda\right)$. There are two Killing vector possibilities: $\xi_{a}=\lambda u_{a}$ and $\xi_{a}=\lambda e_{a}^{(1)}$, where we have chosen $e_{a}^{(1)}$ for convenience. The timelike Killing vector will generate isotropic stress. Consider the spacelike vector $\xi_{a}=\lambda e_{a}^{(1)}$. We have

$$
-(1 / 8 \pi) \Psi|\lambda|\left(g_{a b}-e_{a}^{(1)} e_{b}^{(1)}\right)=\rho u_{a} u_{b}+p_{1} e_{a}^{(1)} e_{b}^{(1)}+p_{2} e_{a}^{(2)} e_{b}^{(2)}+p_{3} e_{a}^{(3)} e_{b}^{(3)}+\left(\frac{\rho-p_{\Sigma}}{2}\right) g_{a b} .
$$

Multiplying by $e_{a}^{(1)}$ we have

$$
p_{1}+\rho=p_{2}+p_{3}
$$

Contracting with $u_{a}$ we find

$$
(1 / 8 \pi) 2 \Psi|\lambda|=\rho+p_{\Sigma} .
$$

The other spatial contractions give

$$
\begin{aligned}
& -(1 / 8 \pi) 2 \Psi|\lambda|=p_{2}-p_{1}-p_{3}+\rho, \\
& -(1 / 8 \pi) 2 \Psi|\lambda|=p_{3}-p_{1}-p_{2}+\rho .
\end{aligned}
$$

Comparing, we must have $p_{2}=p_{3}$, and 


$$
-(1 / 8 \pi) 2 \Psi|\lambda|=-p_{1}+\rho
$$

Combining, we find an anisotropic fluid with density and stress

$$
\begin{gathered}
8 \pi \rho=-\Psi \lambda / 2, \\
8 \pi p_{1}=3 \Psi \lambda / 2, \\
p_{2}=p_{3}=-\rho .
\end{gathered}
$$

\section{B. Anisotropic model 2}

Consider Ricci tensor $R_{a b}=s_{a} s_{b}$. This Ricci tensor model requires that the vector $s^{a}$ be orthogonal to the Killing vector. Again there are two choices for the Killing vector, and in this model it is the timelike Killing vector that generates the anisotropic stress energy. Consider $\xi_{a}$ $=\lambda u_{a}$. Since $s_{a}$ is orthogonal to the Killing vector, $s_{a}$ is spacelike. Choose function $\Phi$ and $s_{a}$ $=\Phi e_{a}^{(1)}$,

$$
\frac{s_{a} s_{b}}{8 \pi}=\frac{\Phi^{2} e_{a}^{(1)} e_{b}^{(1)}}{8 \pi}=\rho u_{a} u_{b}+p_{1} e_{a}^{(1)} e_{b}^{(1)}+p_{2} e_{a}^{(2)} e_{b}^{(2)}+p_{3} e_{a}^{(3)} e_{b}^{(3)}+\left(\frac{\rho-p_{\Sigma}}{2}\right) g_{a b}
$$

Following the same method used in the previous section, we obtain

$$
\begin{gathered}
8 \pi \rho=8 \pi p_{1}=\Phi^{2} / 2, \\
8 \pi p_{2}=8 \pi p_{3}=-\Phi^{2} / 2 .
\end{gathered}
$$

The indices can be relabeled to describe $s_{a}$ lying along $e_{a}^{(2)}$ or $e_{a}^{(3)}$.

\section{SPACE-TIMES FOR THE ANISOTROPIC MATTER DISTRIBUTIONS}

In Sec. IV we examined two anisotropic models. One has a timelike Killing vector and one a spacelike Killing vector. We now find examples of space-times that could contain the anisotropic matter distribution.

\section{A. Timelike Killing vector}

For density and pressures $\rho=p_{1}=-p_{2}=-p_{3}$ consider the metric with function $\chi(z)$ :

$$
\mathrm{d} s^{2}=-e^{2 n \chi} \mathrm{d} t^{2}+\mathrm{d} z^{2}+e^{2 \chi}\left(\mathrm{d} r^{2}+r^{2} \mathrm{~d} \varphi^{2}\right) .
$$

The field equations are

$$
\begin{gathered}
8 \pi \rho=-2 \chi_{, z z}-3 \chi_{, z}^{2}, \\
8 \pi p_{z}=\chi_{, z}^{2}(2 n+1), \\
8 \pi p_{k}=(n+1) \chi_{, z z}+\chi_{, z}^{2}\left(n^{2}+n+1\right)
\end{gathered}
$$

with $p_{k}$ labeling both $p_{r}$ and $p_{\varphi}$. Enforcing the stress relations, one finds the solution

$$
e^{(n+2) \chi}=a z+b
$$

with fluid $\rho=p_{z}=-p_{k}$

$$
8 \pi \rho=\frac{(2 n+1) a^{2}}{(n+2)^{2}(a z+b)^{2}}
$$


and metric

$$
\mathrm{d} s^{2}=-(a z+b)^{2 n /(n+2)} \mathrm{d} t^{2}+\mathrm{d} z^{2}+(a z+b)^{2 /(n+2)}\left(\mathrm{d} r^{2}+r^{2} \mathrm{~d} \varphi^{2}\right) .
$$

For $n=1$, this space-time is conformally flat.

\section{B. Spacelike Killing vector}

A simple space-time whose fluid content has the necessary anisotropic structure is the conformally flat space-time with metric

$$
\mathrm{d} s^{2}=e^{2 a z}\left(-\mathrm{d} t^{2}+\mathrm{d} r^{2}+r^{2} \mathrm{~d} \varphi^{2}+\mathrm{d} z^{2}\right) .
$$

The fluid parameters are easily shown to be

$$
\begin{aligned}
& 8 \pi \rho=-a^{2} e^{-2 a z}, \\
& 8 \pi p_{z}=3 a^{2} e^{-2 a z}, \\
& 8 \pi p_{k}=a^{2} e^{-2 a z},
\end{aligned}
$$

where $p_{k}$ are the radial and $\varphi$-stresses. The negative density does not readily lend itself to a physical description. An interesting space-time that also has the appropriate anisotropic stress relations is the simple lift of the $2+1$ BTZ black hole space-time ${ }^{26}$ describing an infinite black string: 27,28

$$
\mathrm{d} s^{2}=-\left(-m+\Lambda_{3} r^{2}\right) \mathrm{d} t^{2}+\frac{\mathrm{d} r^{2}}{-m+\Lambda_{3} r^{2}}+r^{2} \mathrm{~d} \varphi^{2}+\mathrm{d} z^{2} .
$$

In $2+1$ there is a stress energy

$$
T_{i i}=\Lambda_{3} g_{i i}
$$

with $\Lambda_{3}$ the $2+1$ cosmological constant. When the $z$ coordinate is added the fluid content is

$$
\begin{gathered}
8 \pi \rho=-\Lambda_{3}, \\
8 \pi p_{r}=8 \pi p_{\varphi}=\Lambda_{3}, \\
8 \pi p_{z}=3 \Lambda_{3},
\end{gathered}
$$

which has the required anisotropic stress-energy structure. The relation between the $2+1$ BTZ solution and the $3+1$ black string has been studied by Lemos and Zanchin. ${ }^{29}$ The negative density in this case can be physically motivated from the cosmological constant. It will be of interest to apply the Geroch transform to this $3+1$ BTZ lift and then project back down to $2+1$ to examine the effects on the cosmological fluid.

\section{APPLYING THE GEROCH TRANSFORMATION}

In this section we will use the Geroch formalism described by Eqs. (7)-(10) to add twist to the Killing vectors of our example space-times. The new space-time will be generated and the effect of the transformation on the fluid parameters examined.

\section{A. Timelike Killing vector}

The space-time with a timelike Killing vector that we found had metric

$$
\mathrm{d} s^{2}=-(a z+b)^{2 n /(n+2)} \mathrm{d} t^{2}+\mathrm{d} z^{2}+(a z+b)^{2 /(n+2)}\left(\mathrm{d} r^{2}+r^{2} \mathrm{~d} \varphi^{2}\right) .
$$


The Geroch transform process can be applied to this space-time adding twist to the timelike Killing vector and vorticity to the fluid. From Eqs. (7) to (9) we have

$$
\begin{gathered}
\xi_{a}^{(t)} \xi_{(t)}^{a}=\lambda=-(a z+b)^{2 n /(n+2)}, \\
F=\cos ^{2}(\delta)+\lambda^{2} \sin ^{2}(\delta), \\
\alpha_{\varphi, r}-\alpha_{r, \varphi}=-\frac{2 n a r}{n+2} \eta_{t r \varphi z}, \\
\alpha_{\varphi}=-\frac{n a r^{2}}{n+2}+\alpha_{0}
\end{gathered}
$$

where $\eta_{t r \varphi z}=1$. The new metric is

$$
\mathrm{d} s^{2}=-\frac{(a z+b)^{2 n /(n+2)}}{F}\left[\mathrm{~d} t+\sin (2 \delta) \alpha_{\varphi} \mathrm{d} \varphi\right]^{2}+F \mathrm{~d} z^{2}+F(a z+b)^{2 /(n+2)}\left(\mathrm{d} r^{2}+r^{2} \mathrm{~d} \varphi^{2}\right) .
$$

The fluid parameters in this space-times are the original parameters scaled by $F$ :

$$
\begin{gathered}
\rho^{\prime}=\rho / F, \\
p_{i}^{\prime}=p_{i} / F .
\end{gathered}
$$

The fluid has acquired vorticity along the $z$ axis

$$
\omega^{(z)}=\frac{2 n a \sin (2 \delta)}{(n+2) F^{2}}(a z+b)^{(n-2) /(n+2)} .
$$

The projective transform on the Killing parameters that generates the new space-time has two fixed points. For the case where the initial space is twist free, the fixed points of the projective transform are $\lambda= \pm 1$. For this example, $F=1$ at the fixed points, and the fluid parameters are the same in both the seed and transformed space-times.

\section{B. Spacelike Killing vector}

In this example, we wish to add twist to a spacelike Killing vector. We will consider the lift of the BTZ metric as the seed spacetime. The seed metric is

$$
\mathrm{d} s^{2}=-\left(-m+\Lambda_{3} r^{2}\right) \mathrm{d} t^{2}+\frac{\mathrm{d} r^{2}}{-m+\Lambda_{3} r^{2}}+r^{2} \mathrm{~d} \varphi^{2}+\mathrm{d} z^{2} .
$$

There are two Killing vector choices: $\xi_{a}^{(\varphi)}$ or $\xi_{a}^{(z)}$. We will work with the $\varphi$-Killing vector and assume that the metric remains independent of the $z$-coordinate. From Eq. (7) we have

$$
\begin{gathered}
\xi_{a}^{(\varphi)} \xi_{(\varphi)}^{a}=\lambda=r^{2}, \\
\alpha_{t, z}-\alpha_{z, t}=2 \eta_{t z r \varphi}\left(-m+\Lambda_{3} r^{2}\right) .
\end{gathered}
$$

Calculating $\alpha_{a}$ and $\eta_{a}$ we have

$$
\begin{aligned}
& \alpha_{z}=2\left(m-\Lambda_{3} r^{2}\right) t+\alpha_{0}, \\
& \eta_{a}=\xi_{a}^{(\varphi)} / \lambda+\sin (2 \delta) \alpha_{a},
\end{aligned}
$$


with $F=\cos ^{2}(\delta)+r^{4} \sin ^{2}(\delta)$. The new $3+1$ metric is

$$
\mathrm{d} s^{2}=F\left[-\left(-m+\Lambda_{3} r^{2}\right) \mathrm{d} t^{2}+\frac{\mathrm{d} r^{2}}{-m+\Lambda_{3} r^{2}}+\mathrm{d} z^{2}\right]+\frac{r^{2}}{F}\left[\mathrm{~d} \varphi+\alpha_{z} \sin (2 \delta) \mathrm{d} z\right]^{2} .
$$

This black string solution is an anisotropic member of the cylindrical black hole solution family discussed by Lemos. ${ }^{30}$

Using $\delta=\pi / 2$, it is easy to see the effect of the transform on the fluid parameters. For this value, the new $3+1$ space-time becomes

$$
\mathrm{d} s^{2}=r^{4}\left[-\left(-m+\Lambda_{3} r^{2}\right) \mathrm{d} t^{2}+\frac{d r^{2}}{-m+\Lambda_{3} r^{2}}+\mathrm{d} z^{2}\right]+\frac{1}{r^{2}} \mathrm{~d} \varphi^{2}
$$

with a fluid content

$$
\begin{gathered}
8 \pi \rho=-\Lambda_{3} / r^{4}, \\
8 \pi p_{r}=\Lambda_{3} / r^{4}, \\
8 \pi p_{\varphi}=9 \Lambda_{3} / r^{4}, \\
8 \pi p_{z}=3 \Lambda_{3} / r^{4} .
\end{gathered}
$$

The new general space-time can be projected back into $2+1$ with the result

$$
\begin{gathered}
h_{a b}=g_{a b}-\xi_{a}^{(z)} \xi_{b}^{(z)} / \lambda_{z}, \\
h_{a b} \mathrm{~d} x^{a} \mathrm{~d} x^{b}=F\left[-\left(-m+\Lambda_{3} r^{2}\right) \mathrm{d} t^{2}+\frac{\mathrm{d} r^{2}}{-m+\Lambda_{3} r^{2}}\right]+\frac{r^{2}}{F} \mathrm{~d} \varphi^{2},
\end{gathered}
$$

which can be written as

$$
\begin{aligned}
h_{a b} \mathrm{~d} x^{a} \mathrm{~d} x^{b}= & -\left(-m+\Lambda_{3} r^{2}\right) \mathrm{d} t^{2}+\frac{\mathrm{d} r^{2}}{-m+\Lambda_{3} r^{2}}+r^{2} \mathrm{~d} \varphi^{2}+\left(r^{4}-1\right) \\
& \times \sin ^{2}(\delta)\left[-\left(-m+\Lambda_{3} r^{2}\right) \mathrm{d} t^{2}+\frac{\mathrm{d} r^{2}}{-m+\Lambda_{3} r^{2}}+\frac{r^{2}}{1+\left(r^{4}-1\right) \sin ^{2}(\delta)} \mathrm{d} \varphi^{2}\right] .
\end{aligned}
$$

For $\delta=0$ the original $2+1 \mathrm{BTZ}$ space-time is recovered. It is also recovered at the $r^{2}=1$ fixed point. The fluid content of the $2+1$ space-time is

$$
\begin{gathered}
8 \pi \rho=\frac{-\Lambda_{3}}{F}+\frac{2 r^{2} \sin ^{2}(\delta)}{F^{3}}\left\{m F+\left(-m+\Lambda_{3} r^{2}\right)\left[7 \cos ^{2}(\delta)-r^{4} \sin ^{2}(\delta)\right]\right\}, \\
8 \pi p_{r}=\frac{\Lambda_{3}}{F}-\frac{2 r^{2} \sin ^{2}(\delta)}{F^{3}}\left\{m F+2 r^{4} \sin ^{2}(\delta)\left(-m+\Lambda_{3} r^{2}\right)\right\}, \\
8 \pi p_{\varphi}=\frac{\Lambda_{3}}{F}+\frac{2 r^{2} \sin ^{2}(\delta)}{F^{3}}\left\{2 m F+\left(-m+\Lambda_{3} r^{2}\right)\left[5 \cos ^{2}(\delta)+r^{4} \sin ^{2}(\delta)\right]\right\} .
\end{gathered}
$$

The original BTZ solution described a black hole of mass $m$ surrounded by a cosmological fluid with parameters $8 \pi \rho=-\Lambda_{3}, 8 \pi p_{r}=8 \pi p_{\varphi}=\Lambda_{3}$. From Eq. (54) it is clear that the cosmological fluid is still present but scaled by $F$, and that in addition a new fluid has been added. For $\delta$ $=\pi / 2$, for example, the fluid parameters are 


$$
\begin{gathered}
8 \pi \rho=\left(4 m-3 r^{2} \Lambda_{3}\right) / r^{6}, \\
8 \pi p_{r}=\left(2 m-3 r^{2} \Lambda_{3}\right) / r^{6}, \\
8 \pi p_{\varphi}=\left(2 m+3 r^{2} \Lambda_{3}\right) / r^{6} .
\end{gathered}
$$

At infinity, the new solution describes an empty vacuum in contrast to the cosmological vacuum found in the BTZ asymptotic limit. The original BTZ solution had constant negative curvature making it locally isometric to AdS while the new space-time has a nonconstant Ricci scalar

$$
R=-\frac{6 \Lambda_{3}}{F}+\left(-m+\Lambda_{3} r^{2}\right) \frac{8 r^{2} \sin ^{2}(\delta) \cos ^{2}(\delta)}{F^{3}} .
$$

\section{DISCUSSION}

We have shown that the simple one parameter Ehlers-Geroch transform can be applied to space-times with anisotropic matter content for two different stress-energy situations. The formalism broadens the way in which anisotropy can be introduced and studied with relation to the Killing symmetry of the space-time. The formalism was applied to the simple lift of the BTZ solution and when the new $3+1$ solution was projected back to $2+1$, a different static $2+1$ solution was obtained. It describes a $2+1$ black hole with a horizon at the same position as the original BTZ horizon but with an additional fluid atmosphere. The asymptotic structure of the two solutions is very different. This result suggests that it would be useful to develop the formalism in dimensions higher than $3+1$, and use the projection technique to generate and study the resulting anisotropic $3+1$ solutions. Another generalization which could prove interesting is to broaden the fixed point structure of the projective transform. The fixed points described by Eq. (6) are $\lambda$ $= \pm 1$, as discussed previously. When using the Ehlers-Geroch method with a spacelike Killing vector, generalizing the projective transform by placing the fixed points at $\lambda=L^{2}$ offers a better chance of interpreting the fixed points and would broaden the applicability of the method.

In summary, the solution generating method developed here preserves specific Killing symmetries while creating new anisotropic solutions. These solutions may be useful for investigating relativistic behavior at the density extremes.

\footnotetext{
${ }^{1}$ J. Ehlers, in Les Théories Relativistes de la Gravitation, edited by A. Lichnerowicz and M. A. Tonnelat (Colloques Internationaux C.N.R.S. No. 91, Paris, 1962).

${ }^{2}$ B. K. Harrison, J. Math. Phys. 9, 1744 (1968).

${ }^{3}$ R. Geroch, J. Math. Phys. 12, 918 (1971).

${ }^{4}$ R. Geroch, J. Math. Phys. 13, 394 (1972).

${ }^{5}$ W. Kinnersley, J. Math. Phys. 14, 651 (1973).

${ }^{6}$ V. A. Belinski, Sov. Phys. JETP 50, 623 (1979).

${ }^{7}$ H. Stephani, J. Math. Phys. 29, 1650 (1988).

${ }^{8}$ I. Racz and J. Zsigrai, Class. Quantum Grav. 13, 2783 (1996).

${ }^{9}$ L. Herrera and N. O. Santos, Phys. Rep. 286, 53 (1997).

${ }^{10}$ R. L. Bowers and E. P. T. Liang, Astrophys. J. 188, 657 (1974).

${ }^{11}$ E. S. Corchero, Class. Quantum Grav. 15, 3645 (1998).

${ }^{12}$ A. Das, N. Tariq, D. Aruliah, and T. Biech, J. Math. Phys. 38, 4202 (1998).

${ }^{13}$ A. Das and S. Kloster, Phys. Rev. D 62, 104002 (2000).

${ }^{14}$ H. Hernandez, L. A. Nunez, and U. Percoco, Class. Quantum Grav. 16, 871 (1999).

${ }^{15}$ L. Herrera, A. DiPrisco, J. Ospino, and E. Fuenmayor, J. Math. Phys. 42, 2129 (2001).

${ }^{16}$ E. N. Glass and J. P. Krisch, Phys. Rev. D 57, R5945 (1998).

${ }^{17}$ T. Harko and M. K. Mak, J. Math. Phys. 41, 4752 (2000).

${ }^{18}$ D. J. McManus and A. A. Coley, Class. Quantum Grav. 11, 2045 (1994).

${ }^{19}$ R. J. vandenHoogen and A. A. Coley, Class. Quantum Grav. 12, 2335 (1995).

${ }^{20}$ M. Giovannini, Phys. Rev. D 59, 123518 (1999).

${ }^{21}$ T. Rainsford, Gen. Relativ. Gravit. 33, 1047 (2001).

${ }^{22}$ J. H. Jeans, Mon. Not. R. Astron. Soc. 82, 122 (1922).

${ }^{23}$ P. S. Letelier, Phys. Rev. D 22, 807 (1980).

${ }^{24} \mathrm{D}$. Garfinkle (private communication).

${ }^{25}$ D. Garfinkle, E. N. Glass, and J. P. Krisch, Gen. Relativ. Gravit. 29, 467 (1997).
} 
${ }^{26}$ M. Banados, C. Teitelboim, and J. Zanelli, Phys. Rev. Lett. 69, 1849 (1992).

${ }^{27}$ N. Kaloper, Phys. Rev. D 48, 4658 (1993).

${ }^{28}$ J. S. F. Chan and B. Mann, Phys. Rev. D 51, 5428 (1995).

${ }^{29}$ J. P. S. Lemos and V. Zanchin, Phys. Rev. D 53, 4684 (1996).

${ }^{30}$ J. P. S. Lemos, Phys. Lett. B 353, 46 (1995). 